Studies of the structure and growth mode of dotriacontane films by synchrotron $\mathrm{x}$-ray scattering and molecular dynamics simulations

This article has been downloaded from IOPscience. Please scroll down to see the full text article.

2004 J. Phys.: Condens. Matter 16 S2905

(http://iopscience.iop.org/0953-8984/16/29/005)

View the table of contents for this issue, or go to the journal homepage for more

Download details:

IP Address: 128.206.162.204

The article was downloaded on 08/10/2010 at 19:15

Please note that terms and conditions apply. 


\title{
Studies of the structure and growth mode of dotriacontane films by synchrotron $x$-ray scattering and molecular dynamics simulations
}

\author{
H Mo ${ }^{1}$, S Trogisch ${ }^{1}$, H Taub ${ }^{1}$, S N Ehrlich ${ }^{2}$, U G Volkmann ${ }^{3}$, \\ F Y Hansen ${ }^{4}$ and M Pino ${ }^{3}$ \\ ${ }^{1}$ Department of Physics and Astronomy and University of Missouri Research Reactor, \\ University of Missouri-Columbia, Columbia, MO 65211, USA \\ ${ }^{2}$ National Synchrotron Light Source, Brookhaven National Laboratory, Upton, NY 11973, USA \\ ${ }^{3}$ Facultad de Física, Pontificia Universidad Católica de Chile, Santiago 22, Chile \\ ${ }^{4}$ Department of Chemistry, Technical University of Denmark, IK 207 DTU, \\ DK-2800 Lyngby, Denmark
}

Received 4 May 2004

Published 9 July 2004

Online at stacks.iop.org/JPhysCM/16/S2905

doi:10.1088/0953-8984/16/29/005

\begin{abstract}
We report on synchrotron $\mathrm{x}$-ray scattering experiments and molecular dynamics simulations of the structure and growth mode of dotriacontane $\left(n-\mathrm{C}_{32} \mathrm{H}_{66}\right.$ or C32) films adsorbed on $\mathrm{Ag}(111)$ and $\mathrm{SiO}_{2}$-coated $\mathrm{Si}(100)$ substrates. On the $\mathrm{SiO}_{2}$ surface, the x-ray measurements confirm a structural model of the solid film inferred from high-resolution ellipsometry measurements in which one or two layers of C32 adsorb with the long axis of the molecule oriented parallel to the interface followed by a monolayer in which the molecules have a perpendicular orientation. At higher C32 coverages, preferentially oriented bulk particles nucleate, consistent with a Stranski-Krastanov growth mode. On the $\operatorname{Ag}(111)$ surface, we again observe one or two layers of the 'parallel' film but no evidence of the perpendicular monolayer before nucleation of the preferentially oriented bulk particles. We compare the experimentally observed structures with molecular dynamics simulations of a multilayer film of the homologous C24 molecule.
\end{abstract}

(Some figures in this article are in colour only in the electronic version)

\section{Introduction}

Films of intermediate-length alkanes $\left(n-\mathrm{C}_{\mathrm{n}} \mathrm{H}_{2 \mathrm{n}+2} ; 20<n<40\right)$ are of interest as prototypes of more complex polymer films used in coatings, adhesives, and electronic devices. Alkane films are also of interest in their own right, since alkanes are the principal constituents of commercial lubricants. A microscopic understanding of the structure, phase transitions, and dynamics of 
alkanes near solid interfaces could potentially lead to significant advances in polymer-based technologies and, in particular, lubrication.

The molecular structure of alkane films adsorbed onto graphite surfaces has been investigated for over 20 years. The first experiments were performed by neutron diffraction on films of shorter alkanes (carbon number $\mathrm{n}=2$, 4, and 6) deposited from the vapour phase onto high-surface-area substrates (Grafoil) as reviewed in [1]. Subsequently, structural studies of short-alkane films ( $n=4,6$, and 7) have been extended to synchrotron x-ray scattering experiments on films vapour-deposited on single-crystal $\operatorname{Ag}(111)$ substrates in ultrahigh vacuum (UHV) [2]. There have also been neutron diffraction experiments on films of intermediate-length alkanes $(n=24$ and 32) deposited both from vapour phase and from solution onto Grafoil substrates [3, 4].

More recently, we have begun to investigate by both high-resolution ellipsometry [5] and synchrotron $\mathrm{x}$-ray specular reflectivity [6] the structure of dotriacontane films $(\mathrm{n}=32$, denoted C32) deposited from solution onto $\mathrm{SiO}_{2}$-coated $\mathrm{Si}(100)$ substrates. These measurements have revealed a previously unobserved growth mode of alkane films on a solid substrate: in the first one or two C32 layers adjacent to the substrate, the molecules orient with their long axis parallel to the surface. This 'parallel' film is followed by a monolayer in which the molecules adsorb with their long axis perpendicular to the interface. At still higher coverages, preferentially oriented bulk particles nucleate, indicating a Stranski-Krastanov growth mode.

Since the 'perpendicular' monolayer was not observed in studies of films of shorter $n$ alkane molecules grown from the vapour phase on a $\operatorname{Ag}(111)$ surface [2], it is of interest to ask such questions as whether the growth mode of solid alkane films depends on the length of the molecules, the choice of substrate, and the deposition method. To address these issues, we have undertaken $\mathrm{x}$-ray specular reflectivity measurements on $\mathrm{C} 32$ films grown from the vapour phase on single-crystal $\mathrm{Ag}(111)$ substrates in UHV. These experiments are part of a larger programme to study the structure of both solid and fluid alkane films adsorbed on wellcharacterized metal surfaces with the aim of better understanding lubrication phenomena at the microscopic level.

\section{Methods and materials}

For this study, the C32 films were grown in situ using a specially designed UHV chamber. Vapour from the $\mathrm{C} 32$ liquid reservoir in a Knudsen cell effused through a small aperture $(0.5 \mathrm{~mm}$ diameter) onto the $\mathrm{Ag}(111)$ substrate $(10 \mathrm{~mm}$ diameter) located at a distance of $2.5 \mathrm{~cm}$ from the aperture. During deposition, the Knudsen cell was held at a temperature of $73{ }^{\circ} \mathrm{C}$. For monolayer coverage, the $\mathrm{Ag}(111)$ substrate was held at $106^{\circ} \mathrm{C}$ to prevent thicker film growth. The multilayer was grown with the substrate at $42{ }^{\circ} \mathrm{C}$, exposing it to the $\mathrm{C} 32$ vapour for $20 \mathrm{~min}$.

We conducted the $\mathrm{x}$-ray reflectivity measurements at the 6-IDB station of MUCAT at the Advanced Photon Source, Argonne National Laboratory. Specular reflectivity curves were obtained by integrating the intensity of individual 'rocking' curves taken at closely spaced intervals along the specular rod (wavevector transfer $Q<2.5 \AA^{-1}$ ). After making corrections for beam spillover, other geometrical effects, and $Q$-resolution, the curves were fitted with an atomic model that included contributions from both the Ag crystal and the alkane layers [2]. From the fit, parameters such as the height of the alkane layers and their density were obtained.

\section{Results}

The X-ray specular curves taken at room temperature for a monolayer and a 'multilayer' sample are shown in figures 1 and 2, respectively. The solid symbols are data points and the solid lines are the best fits to the model that assumed the first $N$ molecular layers of the film to be complete 

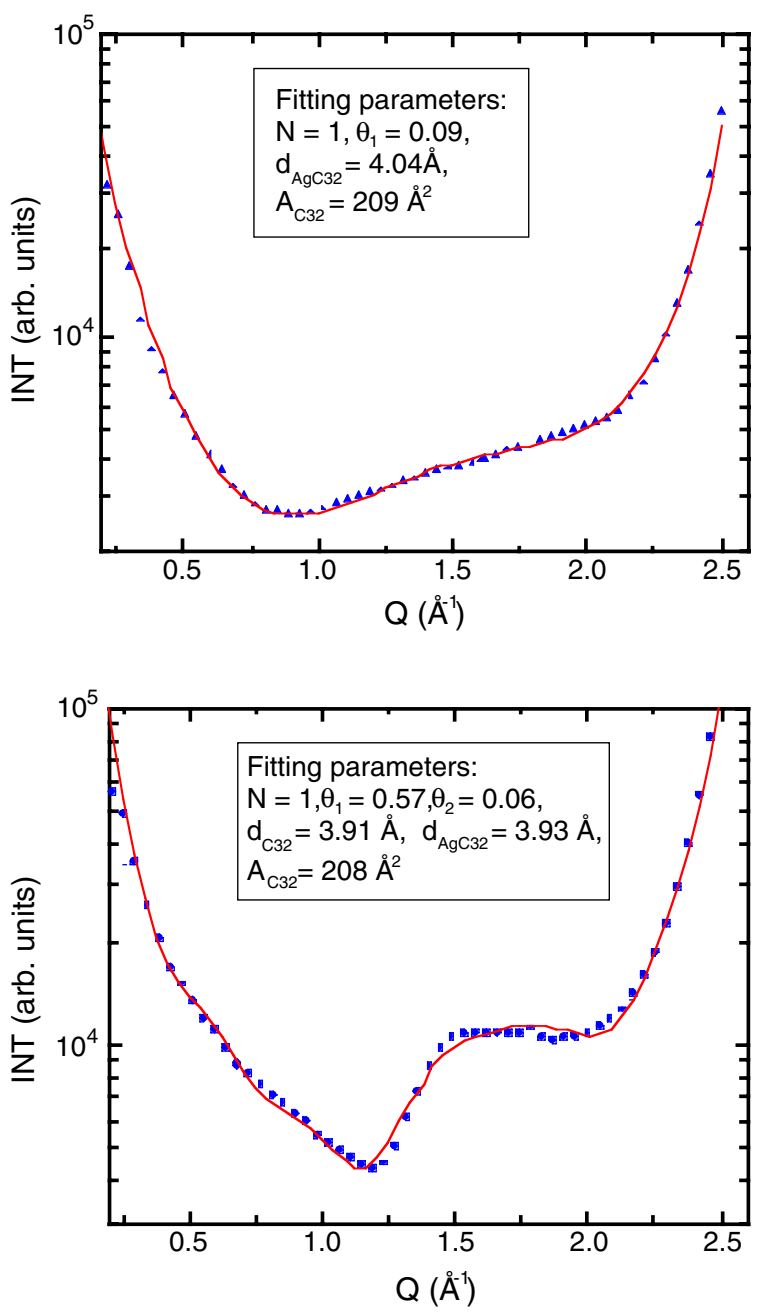

Figure 1. Integrated specular reflectivity scans as a function of wavevector transfer at room temperature for a $\mathrm{C} 32$ monolayer vapour-deposited on the $\mathrm{Ag}(111)$ surface.

Figure 2. Integrated specular reflectivity scans as a function of wavevector transfer at room temperature for a C32 'multilayer' vapour-deposited on the $\mathrm{Ag}(111)$ surface.

followed by up to three partial layers with fractional occupancy $\theta_{1}, \theta_{2}, \theta_{3}$, respectively (note that the subscript indicates the number of partial layers following $N$ complete layers). The C32 molecules were taken to be in the all-trans configuration.

In the case of the monolayer, the best fit is obtained with the C32 carbon skeletal plane parallel to the substrate, whereas parallel and perpendicular orientations yielded comparable fits for the 'multilayer' film. The monolayer fit gives an area of $A_{\mathrm{C} 32}=209 \AA^{2}$ per molecule, a height of the carbon skeletal plane above the $\operatorname{Ag}(111)$ surface of $d_{\mathrm{AgC} 32}=4.04 \AA$, and a second layer occupancy of $\theta_{1} \sim 9 \%$.

Assuming a parallel orientation of the molecules, the fit to the 'multilayer' gives about the same area per molecule $\left(A_{\mathrm{C} 32}=208 \AA^{2}\right)$ and first C32 layer height $\left(d_{\mathrm{AgC} 32}=3.93 \AA\right)$ as for the monolayer. Although we estimated the amount of C32 deposited on the substrate for the 'multilayer' sample to be greater than that required to grow four layers of parallel-oriented molecules, the best fit gives fractional occupancies $\theta_{1} \sim 60 \%$ and $\theta_{2} \sim 6 \%$ of the second and third layers, respectively. It also gives a distance of $d_{\mathrm{C} 32}=3.91 \AA$ between the $\mathrm{C} 32$ layers.

To investigate the possibility of growing thicker C32 films on the $\mathrm{Ag}(111)$ substrate, we increased both the exposure time to the C32 vapour and the temperature of the Knudsen cell. 


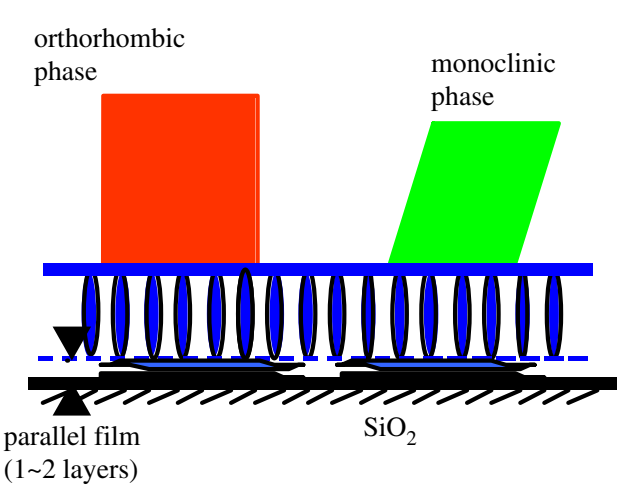

Figure 3. Model proposed for the growth of C32 films from solution on a $\mathrm{SiO}_{2}$ surface.

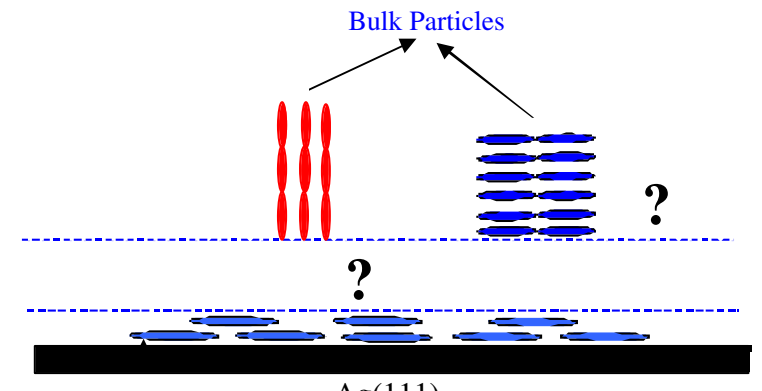

$\operatorname{Ag}(111)$
Figure 4. Model proposed for the growth of vapour-deposited $\mathrm{C} 32$ films on an $\mathrm{Ag}(111)$ surface.

In these cases (not shown here), we observed Bragg peaks from preferentially oriented bulk particles consistent with the nucleation of an orthorhombic phase as found for C32 deposited onto the $\mathrm{SiO}_{2}$-coated $\mathrm{Si}(100)$ substrates [6].

\section{Discussion}

It is interesting to compare the growth mode of $\mathrm{C} 32$ on $\mathrm{Ag}(111)$ with that on the $\mathrm{SiO}_{2} / \mathrm{Si}(100)$ substrate. In both cases, we have inferred that one to two layers of C32 adsorb first with the long molecular axis oriented parallel to the surface as shown in figures 3 and 4. Also, for both substrates at sufficiently high coverage, preferentially oriented bulk particles coexist with the film, as in Stranski-Krastanov growth. On the $\mathrm{SiO}_{2}$ surface, two series of bulk Bragg peaks are observed, corresponding to particles of a surface stabilized orthorhombic phase as well as the previously reported monoclinic structure [7], both oriented with the $a b$-plane parallel to the film plane, as shown schematically in figure 3 [6].

The inferred orientation of the bulk particles on the $\mathrm{Ag}(111)$ surface is shown schematically in figure 4. Again, we find evidence of an orthorhombic phase with particles oriented such that the long axis of the molecules is perpendicular to the surface. In addition, we observe a Bragg peak corresponding to a $d$-spacing of $4.16 \AA$, consistent with a particle orientation rotated $90^{\circ}$ such that the long molecular axis is parallel to the surface. Such a particle has been labelled with a question mark in figure 4 to indicate uncertainty as to whether it has an orthorhombic or monoclinic structure.

For C32 film growth on the $\mathrm{Ag}(111)$ substrate, we do not observe Kiessig fringes with a period consistent with the presence of a $\mathrm{C} 32$ monolayer with molecules oriented perpendicular to the surface as we did for growth on the $\mathrm{SiO}_{2}$ surface. We have indicated this by placing a question mark in the region between the parallel layers and the bulk particles in figure 4 . 


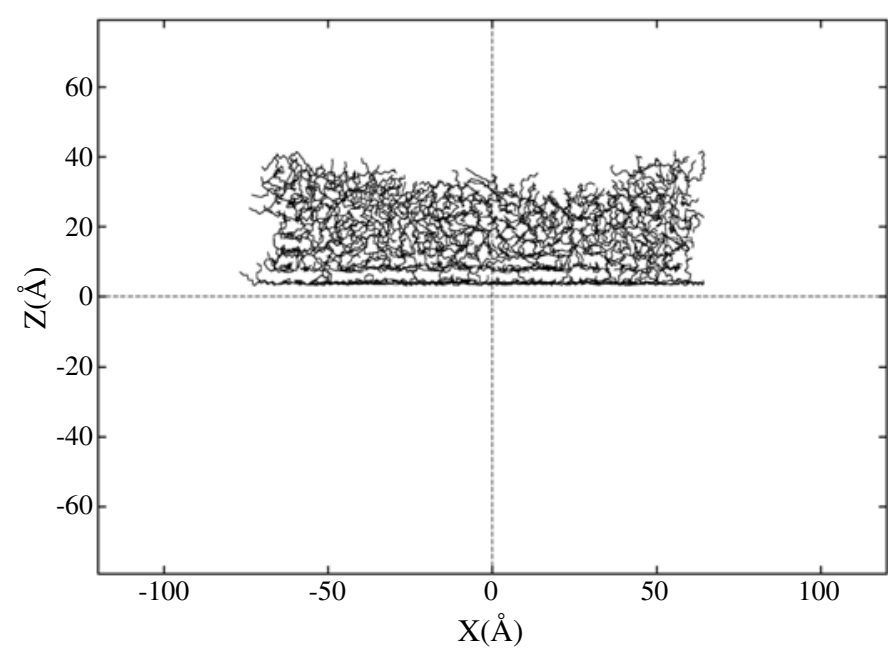

Figure 5. MD simulation of $224 \mathrm{C} 24$ molecules adsorbed onto a graphite substrate at $370 \mathrm{~K}: x-z$ view of the instantaneous configuration of the system after it was allowed to evolve for $14 \mathrm{~ns}$.

Also, our fits to the reflectivity curves of the multilayer samples (including those not presented here) indicate a much smaller film thickness than that estimated from the vapour deposition times, assuming about the same sticking coefficient found for shorter alkanes [2]. We note, in addition, that the quality of the multilayer fits is not as good as for the monolayer in figure 1 .

To aid in interpreting our x-ray scattering results, we have begun extensive molecular dynamics (MD) simulations of film growth of intermediate-length alkanes on a solid substrate. In the first exploratory simulations, we used the smaller C24 molecule (carbon number $\mathrm{n}=24$ ) in the unified-atom approximation [8] in order to reduce the computation time and a graphite basal plane-surface as in previous simulations [4]. To illustrate our results, we describe simulations of a multilayer system at temperatures of 370 and $300 \mathrm{~K}$, above and below the bulk melting point of $324 \mathrm{~K}$, respectively. The simulation cell contained a total of $224 \mathrm{C} 24$ molecules placed above the graphite surface in 7 layers of 32 molecules each. Initially, the molecules had their long axis (defined as the principal axis with the smallest moment of inertia) aligned parallel to the surface in the same configuration that the simulations had given for a crystalline monolayer on graphite [4].

Figure 5 shows an $x-z$ view ( $z$-axis perpendicular to the graphite surface) of the instantaneous configuration of the fluid film at $370 \mathrm{~K}$ after it was allowed to evolve for $14 \mathrm{~ns}$. The top layer of carbon atoms in the graphite surface is at $z=0$. Despite the fluid state of the film, we see in figure 5 one to two layers of C24 nearest the graphite substrate still aligned with their long axis parallel to the surface. Above these parallel layers are randomly oriented molecules containing many gauche defects characteristic of the bulk fluid.

The system was then cooled instantaneously from 370 to $300 \mathrm{~K}$ and propagated for an additional $17 \mathrm{~ns}$. In figure 6, we see that the structure is much more ordered than at the higher temperature. In the crystallization process, the average end-to-end distance of the molecules increased from about $18 \AA$ at $370 \mathrm{~K}$ to $27 \AA$ at $300 \mathrm{~K}$, reflecting a large reduction in the number of gauche defects in the chains. In agreement with the growth mode inferred experimentally for the $\operatorname{Ag}(111)$ surface (figure 4), the simulation shows nearly two complete layers of molecules oriented with their long axis parallel to the surface. The structure of these parallel layers resembles the crystalline monolayer structure on graphite simulated earlier [4]. 


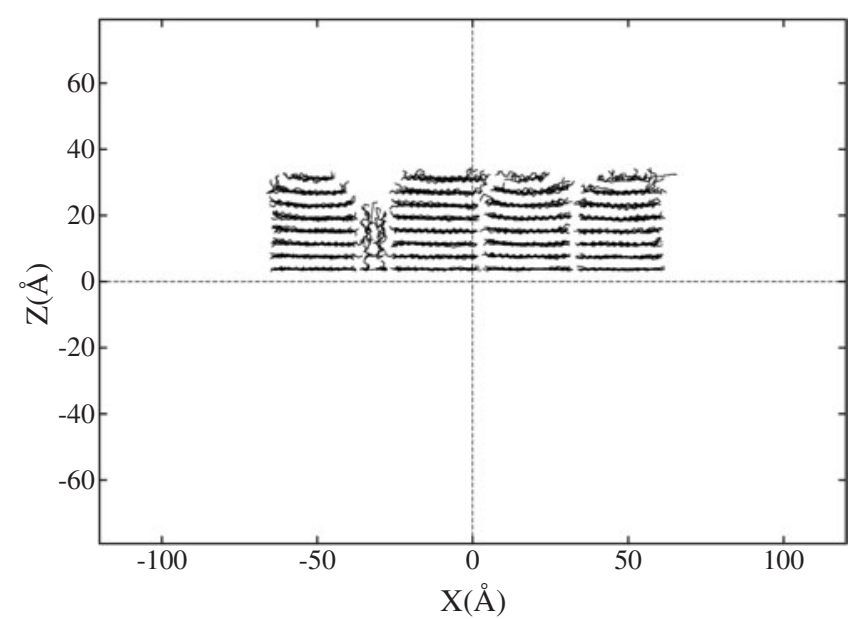

Figure 6. MD simulation of $224 \mathrm{C} 24$ molecules adsorbed onto a graphite substrate at $370 \mathrm{~K}: x-z$ view of the instantaneous configuration of the system after it was equilibrated at $370 \mathrm{~K}$, cooled instantaneously to $300 \mathrm{~K}$, and then allowed to evolve for an additional $17 \mathrm{~ns}$.

Above the first two parallel layers, we observe regions where there are additional layers of parallel molecules as well as disordered regions. The disordered regions may explain why fits of $N$-layer models as in figure 2 yielded a smaller film thickness than that estimated from the vapour deposition times. Further simulations with a larger cell, more molecules, and longer run times will be required to determine whether the more ordered regions above the parallel layers in figure 6 actually represent embryos of bulk particles in which the molecules are oriented parallel to the surface, as shown in figure 4. They may also reveal whether particles in which the molecules are oriented perpendicular to the surface (see figure 4) will emerge from the more disordered regions. Other simulations are underway in which the molecules are initially placed in a gas phase above the graphite surface in order to better simulate a vapour-deposition experiment.

\section{Acknowledgments}

This work was support by US National Science Foundation under Grant Nos. DMR-9802476 and DMR-0109057. The Midwest Universities Collaborative Access Team (MUCAT) sector at the Advanced Photon Source (APS) is supported by the US Department of Energy (DOE), Office of Science, Office of Basic Energy Sciences (BES), through Ames Laboratory under Contract No. W-7405-Eng-82. Use of the APS was supported by the DOE BES under Contract No. W-31-109-ENG-38.

\section{References}

[1] Taub H 1988 The Time Domain in Surface and Structural Dynamics (NATO Advanced Study Institutes, Series C vol 228) ed G J Long and F Grandjean (Dordrecht: Kluwer) p 467

[2] Wu Z, Ehrlich S N, Matthies B, Herwig K W, Dai P, Volkmann U G, Hansen F Y and Taub H 2001 Chem. Phys. Lett. 348168

[3] Herwig K W, Matthies B and Taub H 1995 Phys. Rev. Lett. 753154

[4] Hansen F Y, Herwig K W, Matthies B and Taub H 1999 Phys. Rev. Lett. 832362

[5] Volkmann U G, Pino M, Altamirano L A, Taub H and Hansen F Y 2002 J. Chem. Phys. 1162107

[6] Mo H, Taub H, Volkmann U G, Pino M, Ehrlich S N, Hansen F Y, Lu E and Miceli P 2003 Chem. Phys. Lett. 37799

[7] Craig S R, Hastle G P, Roberts K J and Sherwood J N 1994 J. Mater. Chem. 4977

[8] Ryckaert J P, Cicotti G and Berendsen H J C 1977 J. Comput. Phys. 23327 